PROCEEDINGS OF THE

AMERICAN MATHEMATICAL SOCIETY

Volume 126, Number 9, September 1998, Pages 2663-2675

S $0002-9939(98) 04335-4$

\title{
SMOOTH RANK ONE PERTURBATIONS OF SELFADJOINT OPERATORS
}

\author{
S. HASSI, H. S. V. DE SNOO, AND A. D. I. WILLEMSMA
}

(Communicated by Palle E. T. Jorgensen)

\begin{abstract}
Let $A$ be a selfadjoint operator in a Hilbert space $\mathfrak{H}$ with inner product $[\cdot, \cdot]$. The rank one perturbations of $A$ have the form $A+\tau[\cdot, \omega] \omega, \tau \in \mathbb{R}$, for some element $\omega \in \mathfrak{H}$. In this paper we consider smooth perturbations, i.e. we consider $\omega \in \operatorname{dom}|A|^{k / 2}$ for some $k \in \mathbb{N} \cup\{0\}$. Function-theoretic properties of their so-called $Q$-functions and operator-theoretic consequences will be studied.
\end{abstract}

\section{INTRODUCTION}

Let $A$ be a selfadjoint operator in a Hilbert space $\mathfrak{H}$ with inner product $[\cdot, \cdot]$. For a nontrivial element $\omega \in \mathfrak{H}$ the rank one perturbations of $A$ are defined by

$$
A(\tau)=A+\tau[\cdot, \omega] \omega, \quad \tau \in \mathbb{R},
$$

cf. [4]. Let $S$ be the restriction of $A$ to the orthogonal complement of $\operatorname{span}\{\omega\}$ :

$$
\operatorname{dom} S=\{h \in \operatorname{dom} A:[h, \omega]=0\} .
$$

Then $S$ is a nondensely defined, closed symmetric operator with defect numbers $(1,1)$. Clearly, the perturbations $A(\tau)$ in $(1.1)$ are selfadjoint extensions of $S$ and $\operatorname{dom} A(\tau)=\operatorname{dom} A, \tau \in \mathbb{R}$. Since all selfadjoint extensions of $S$ are parametrized over $\mathbb{R} \cup\{\infty\}$, one selfadjoint extension of $S$ is not of the form (1.1). It is given by

$$
A(\infty)=S \dot{+}(\{0\} \oplus \operatorname{span}\{\omega\}),
$$

which is a selfadjoint relation (multivalued operator), whose multivalued part mul $A$ is given by mul $A=\operatorname{span}\{\omega\}$. In fact, $A(\infty)$ is the generalized Friedrichs extension of $S$ [3], [6], [8]. There is a more general interpretation of (1.1) by allowing $\omega$ to belong to the scale spaces $\mathfrak{H}_{-1}(A)$ and $\mathfrak{H}_{-2}(A)$, associated with $\mathfrak{H}_{+1}(A)=\operatorname{dom}|A|^{\frac{1}{2}}$ and $\mathfrak{H}_{+2}(A)=\operatorname{dom} A$, respectively, [2], [5], [6], [11], [14], [16]. In the present paper our interest is in the spectral properties of smooth perturbations of $A$, i.e. perturbations for which $\omega \in \operatorname{dom}|A|^{k / 2}$ for some $k \in \mathbb{N} \cup\{0\}$.

The main emphasis is on a function-theoretic description of the corresponding $Q$-functions. These functions belong to the class $\mathbf{N}$ of Nevanlinna functions. A subdivision of $\mathbf{N}$ was originated by I.S. Kac [12], [13] and further extended in [9]. In this paper a complete subdivision of $\mathbf{N}$ is presented. We show by means

Received by the editors December 26, 1996 and, in revised form, January 28, 1997.

1991 Mathematics Subject Classification. Primary 47A55, 47A57, 47B25; Secondary 81 Q15.

Key words and phrases. Rank one perturbation, moments, selfadjoint extension, $Q$-function, Friedrichs extension. 
of asymptotic expansions how these subclasses of Nevanlinna functions (and their moments) behave under certain linear fractional transformations. For this purpose we need an extension of asymptotic results due to Hamburger and Nevanlinna; cf. [1, Theorem 3.2.1]. Finally, we connect the function-theoretic results to rank one perturbations.

\section{Preliminaries}

A function $Q(\ell)$ belongs to the class $\mathbf{N}$ of Nevanlinna functions if $Q(\ell)$ is holomorphic on $\mathbb{C} \backslash \mathbb{R}, \overline{Q(\ell)}=Q(\bar{\ell})$, and $\operatorname{Im} Q(\ell) / \operatorname{Im} \ell \geq 0$ for $\ell \in \mathbb{C} \backslash \mathbb{R}$. It is well known that a function $Q(\ell)$ belongs to $\mathbf{N}$ if and only if there exist $\alpha \in \mathbb{R}, \beta \geq 0$, and a nondecreasing function $\sigma(t)$ on $\mathbb{R}$ with $\int_{\mathbb{R}} d \sigma(t) /\left(t^{2}+1\right)<\infty$, such that

$$
Q(\ell)=\alpha+\beta \ell+\int_{\mathbb{R}}\left(\frac{1}{t-\ell}-\frac{t}{t^{2}+1}\right) d \sigma(t)
$$

Clearly, (2.1) implies that

$$
\frac{\operatorname{Im} Q(i y)}{y}=\beta+\int_{\mathbb{R}} \frac{1}{t^{2}+y^{2}} d \sigma(t), \quad y \neq 0 .
$$

A function $Q(\ell)$ belongs to the Kac class $\mathbf{N}_{1}$ if and only if

$$
Q(\ell) \in \mathbf{N} \text { and } \int_{1}^{\infty} \frac{\operatorname{Im} Q(i y)}{y} d y<\infty .
$$

It follows from $\int_{\mathbb{R}}\left(t^{2}+y^{2}\right)^{-1} d y=1 /|t|(\pi / 2-\arctan 1 /|t|), t \neq 0$, that $Q(\ell)$ belongs to $\mathbf{N}_{1}$ if and only if there exist $\gamma \in \mathbb{R}$ and a nondecreasing function $\sigma(t)$ on $\mathbb{R}$ with $\int_{\mathbb{R}} d \sigma(t) /(|t|+1)<\infty$, such that

$$
Q(\ell)=\gamma+\int_{\mathbb{R}} \frac{d \sigma(t)}{t-\ell}
$$

cf. [12], [13]. Observe that the constant $\gamma$ is given by

$$
\gamma=\lim _{y \rightarrow \infty} Q(i y)=\lim _{y \rightarrow \infty} \operatorname{Re} Q(i y)
$$

A function $Q(\ell)$ belongs to the class $\mathbf{N}_{0}$ if and only if

$$
Q(\ell) \in \mathbf{N} \text { and } \sup _{y>0} y \operatorname{Im} Q(i y)<\infty
$$

or equivalently, if there exist $\gamma \in \mathbb{R}$ and a nondecreasing function $\sigma(t)$ on $\mathbb{R}$ with

$$
\int_{\mathbb{R}} d \sigma(t)<\infty
$$

such that (2.3) holds. Clearly, $\mathbf{N}_{0} \subset \mathbf{N}_{1} \subset \mathbf{N}$.

Let the function $Q(\ell)$ belong to $\mathbf{N}$ and fix $\mu \in \mathbb{C} \backslash \mathbb{R}$. For $\tau \in \mathbb{R} \cup\{\infty\}$ we define a linear fractional transformation $Q_{\tau}(\ell)$ of $Q(\ell)$. When $\tau \in \mathbb{R}$ we define

$$
Q_{\tau}(\ell)=\frac{Q(\ell)-\tau(\operatorname{Im} Q(\mu))^{2}}{\tau Q(\ell)+1}=\frac{1}{\tau}-\frac{1+(\tau \operatorname{Im} Q(\mu))^{2}}{\tau^{2}} \frac{1}{\frac{1}{\tau}+Q(\ell)},
$$

and when $\tau=\infty$ we define

$$
Q_{\infty}(\ell)=-\frac{(\operatorname{Im} Q(\mu))^{2}}{Q(\ell)} .
$$

For each $\tau \in \mathbb{R} \cup\{\infty\}$ the function $Q_{\tau}(\ell)$ belongs to $\mathbf{N}$. Moreover, if $Q(\ell)$ belongs to $\mathbf{N}_{1}$ or $\mathbf{N}_{0}$, then for all but one $\tau \in \mathbb{R} \cup\{\infty\}$ the corresponding function $Q_{\tau}(\ell)$ belongs 
to $\mathbf{N}_{1}$ or $\mathbf{N}_{0}$, respectively. The exceptional value of $\tau \in \mathbb{R} \cup\{\infty\}, \tau \neq 0$, is given by $1 / \tau+\gamma=0$, where $\gamma$ is the limit in (2.4); cf. [8]. If $Q(\ell)$ reduces to a real constant $c$, then the exceptional value $\tau$ is given by $1 / \tau+c=0$ and the corresponding linear fractional transform is interpreted as $\infty$. In the rest of this paper we will tacitly exclude this situation. Finally, note that $\beta_{\tau}=\lim _{y \rightarrow \infty} \operatorname{Im} Q_{\tau}(i y) / y$, exists for $\tau \in \mathbb{R} \cup\{\infty\}$ and that if $Q(\ell) \in \mathbf{N}_{0}$, then

$$
\beta_{\tau}=0, \quad \frac{1}{\tau}+\gamma \neq 0, \quad \text { and } \quad \beta_{\tau}=: \beta>0, \quad \frac{1}{\tau}+\gamma=0 .
$$

\section{Subclasses of Nevanlinna functions}

For any function $Q(\ell)$ in $\mathbf{N}$ we define $Q^{[0]}(y)=\frac{\operatorname{Im} Q(i y)}{y}$. If $Q(\ell) \in \mathbf{N}_{0}$ we define

$$
Q^{[2]}(y)=\sup _{y>0} y^{2} Q^{[0]}(y)-y^{2} Q^{[0]}(y) .
$$

According to [9], $Q(\ell)$ belongs to $\mathbf{N}_{-1}$ if and only if

$$
Q(\ell) \in \mathbf{N}_{0} \text { and } \int_{1}^{\infty} Q^{[2]}(y) d y<\infty,
$$

and $Q(\ell)$ belongs to $\mathbf{N}_{-2}$ if and only if

$$
Q(\ell) \in \mathbf{N}_{0} \text { and } \sup _{y>0} y^{2} Q^{[2]}(y)<\infty .
$$

Therefore, $\mathbf{N}_{-2} \subset \mathbf{N}_{-1} \subset \mathbf{N}$. Now we proceed by induction. Assume that $Q(\ell)$ belongs to $\mathbf{N}_{-2 k}$ for some $k \in \mathbb{N} \cup\{0\}$ and that the function $Q^{[2 k]}(y)$ has been given with $\sup _{y>0} y^{2} Q^{[2 k]}(y)<\infty$. Then we define

$$
Q^{[2 k+2]}(y)=\sup _{y>0} y^{2} Q^{[2 k]}(y)-y^{2} Q^{[2 k]}(y) .
$$

The function $Q(\ell)$ belongs to $\mathbf{N}_{-2 k-1}$ if and only if

$$
Q(\ell) \in \mathbf{N}_{-2 k} \text { and } \int_{1}^{\infty} Q^{[2 k+2]}(y) d y<\infty,
$$

and the function $Q(\ell)$ belongs to $\mathbf{N}_{-2 k-2}$ if and only if

$$
Q(\ell) \in \mathbf{N}_{-2 k} \text { and } \sup _{y>0} y^{2} Q^{[2 k+2]}(y)<\infty .
$$

Clearly,

$$
\cdots \subset \mathbf{N}_{-2 k-2} \subset \mathbf{N}_{-2 k-1} \subset \mathbf{N}_{-2 k} \subset \cdots \subset \mathbf{N}_{-2} \subset \mathbf{N}_{-1} \subset \mathbf{N}_{0} .
$$

We give an equivalent description of the classes $\mathbf{N}_{-k}$.

Theorem 3.1. Assume that $Q(\ell) \in \mathbf{N}_{0}$ has the integral representation (2.3) with $\gamma \in \mathbb{R}$ and $\sigma(t)$ as in (2.5). Let $k \in \mathbb{N} \cup\{0\}$. Then $Q(\ell) \in \mathbf{N}_{-k}$ if and only if

$$
\int_{\mathbb{R}}\left(|t|^{k}+1\right) d \sigma(t)<\infty
$$

Proof. We begin with the case of even indices. We claim that

$$
Q(\ell) \in \mathbf{N}_{-2 k} \text { if and only if } \int_{\mathbb{R}}\left(t^{2 k}+1\right) d \sigma(t)<\infty,
$$

in which case

$$
Q^{[2 k+2]}(y)=\int_{\mathbb{R}} \frac{t^{2 k+2}}{t^{2}+y^{2}} d \sigma(t) .
$$


If $k=0,(3.3)$ is clear and (3.4) follows as

$$
Q^{[2]}(y)=\int_{\mathbb{R}} d \sigma(t)-\int_{\mathbb{R}} \frac{y^{2}}{t^{2}+y^{2}} d \sigma(t)=\int_{\mathbb{R}} \frac{t^{2}}{t^{2}+y^{2}} d \sigma(t) .
$$

Now assume that (3.3) and (3.4) hold for some $k \in \mathbb{N} \cup\{0\}$. Then

$$
\sup _{y>0} y^{2} Q^{[2 k+2]}(y)=\int_{\mathbb{R}} t^{2 k+2} d \sigma(t) \quad(\leq \infty) .
$$

Hence, if $Q(\ell) \in \mathbf{N}_{-2 k}$, then

$$
Q(\ell) \in \mathbf{N}_{-2 k-2} \text { if and only if } \int_{\mathbb{R}}\left(t^{2 k+2}+1\right) d \sigma(t)<\infty,
$$

in which case

$$
Q^{[2 k+4]}(y)=\int_{\mathbb{R}} t^{2 k+2} d \sigma(t)-\int_{\mathbb{R}} \frac{t^{2 k+2} y^{2}}{t^{2}+y^{2}} d \sigma(t)=\int_{\mathbb{R}} \frac{t^{2 k+4}}{t^{2}+y^{2}} d \sigma(t) .
$$

Therefore, (3.3) and (3.4) hold with $k \in \mathbb{N} \cup\{0\}$ replaced by $k+1$.

We now take care of the case of odd indices. For $Q(\ell) \in \mathbf{N}_{-2 k},(3.4)$ implies that

$$
\int_{1}^{\infty} Q^{[2 k+2]}(y) d y=\int_{\mathbb{R}} t^{2 k+2} \frac{1}{|t|}\left(\frac{\pi}{2}-\arctan \frac{1}{|t|}\right) d \sigma(t) \quad(\leq \infty) .
$$

Hence, if $Q(\ell) \in \mathbf{N}_{-2 k}$, we conclude that

$$
Q(\ell) \in \mathbf{N}_{-2 k-1} \text { if and only if } \int_{\mathbb{R}}\left(|t|^{2 k+1}+1\right) d \sigma(t)<\infty .
$$

Let $Q(\ell) \in \mathbf{N}_{-k}, k \in \mathbb{N} \cup\{0\}$. Then according to Theorem 3.1 the moments

$$
m_{i}=\int_{\mathbb{R}} t^{i} d \sigma(t), \quad i=0, \ldots, k,
$$

are well defined as absolutely convergent integrals. The following theorem with $k$ even is well known; cf. [1].

Theorem 3.2. Let $Q(\ell)$ be a function in $\mathbf{N}_{0}$ and assume that it has the integral representation (2.3) with $\gamma \in \mathbb{R}$ and $\sigma(t)$ as in (2.5). If $Q(\ell) \in \mathbf{N}_{-k}$ for some $k \in \mathbb{N} \cup\{0\}$, then $Q(\ell)$ has the asymptotic expansion

$$
\ell^{k+1}\left(Q(\ell)-\gamma+\sum_{i=0}^{k} \frac{m_{i}}{\ell^{i+1}}\right)=o(1), \quad \ell \rightarrow \infty,
$$

uniformly for $\delta \leq \arg \ell \leq \pi-\delta$ with any $0<\delta<\frac{1}{2} \pi$. Moreover, if $k$ is odd, the function in the left side of (3.5) belongs to $\mathbf{N}_{1}$.

Proof. For $Q(\ell) \in \mathbf{N}_{-k}$ the moments $m_{i}, i=0, \ldots, k$, are well defined, and

$$
\ell^{k+1}\left(Q(\ell)-\gamma+\sum_{i=0}^{k} \frac{m_{i}}{\ell^{i+1}}\right)=\int_{\mathbb{R}} \frac{t^{k+1}}{t-\ell} d \sigma(t) .
$$

As in [1], it follows that the function in the right side is $o(1)$ as $\ell \rightarrow \infty$. Moreover, for $\ell=i y$, the right side of (3.6) is equal to

$$
\int_{\mathbb{R}} \frac{t^{k+2}}{t^{2}+y^{2}} d \sigma(t)+i \int_{\mathbb{R}} \frac{t^{k+1} y}{t^{2}+y^{2}} d \sigma(t)
$$


Hence, if $k$ is odd, the function in the left side of (3.5) is a Nevanlinna function, which even belongs to $\mathbf{N}_{1}$.

Conversely, the class $\mathbf{N}_{-k}$ can be described in terms of these asymptotic expansions. For $k$ even, the statement of the following result may be found in [1].

Theorem 3.3. Let $k \in \mathbb{N} \cup\{0\}$, and let $\hat{\gamma}$ and $\hat{m}_{i}, i=0, \ldots, k$, be real numbers. Let the function $Q(\ell) \in \mathbf{N}$ have the asymptotic expansion

$$
\ell^{k+1}\left(Q(\ell)-\hat{\gamma}+\sum_{i=0}^{k} \frac{\hat{m}_{i}}{\ell^{i+1}}\right)=o(1),
$$

for $\ell=i y, y \rightarrow \infty$. Then the function in the left side of (3.7) belongs to $\mathbf{N}$ if $k$ is odd. If $k$ is even, or if $k$ is odd and the function in the left side of (3.7) belongs to $\mathbf{N}_{1}$, then $Q(\ell) \in \mathbf{N}_{-k}$ and $\hat{\gamma}=\gamma, \hat{m}_{i}=m_{i}, i=0, \ldots, k$.

Proof. For $k$ even, we refer to [1]. For $k$ odd, (3.7) implies that

$$
\ell^{k}\left(Q(\ell)-\hat{\gamma}+\sum_{i=0}^{k-1} \frac{\hat{m}_{i}}{\ell^{i+1}}\right)=-\frac{\hat{m}_{k}}{\ell}+o\left(\frac{1}{\ell}\right)=o(1), \quad \ell=i y \rightarrow \infty .
$$

As $k-1$ is even, we conclude that $Q(\ell) \in \mathbf{N}_{-k+1}$ and $\hat{\gamma}=\gamma, \hat{m}_{i}=m_{i}, i=$ $0, \ldots, k-1$. Hence, the left side of (3.7) is given by

$$
\ell^{k+1}\left(Q(\ell)-\gamma+\sum_{i=0}^{k-1} \frac{m_{i}}{\ell^{i+1}}\right)+\hat{m}_{k}=\int_{\mathbb{R}} \frac{t^{k} \ell}{t-\ell} d \sigma(t)+\hat{m}_{k} .
$$

For $\ell=i y$ this is equal to

$$
-\int_{\mathbb{R}} \frac{t^{k} y^{2}}{t^{2}+y^{2}} d \sigma(t)+i \int_{\mathbb{R}} \frac{t^{k+1} y}{t^{2}+y^{2}} d \sigma(t)+\hat{m}_{k}
$$

Therefore, for $k$ odd, the function in the left side of (3.7) is a Nevanlinna function. Under the further assumption that the function in the left side of (3.7) belongs to $\mathbf{N}_{1}$, it follows that $\int_{\mathbb{R}}\left(|t|^{k}+1\right) d \sigma(t)<\infty$, so that the moment $m_{k}$ is well defined and $Q(\ell) \in \mathbf{N}_{-k}$. By taking $y \rightarrow \infty$ in (3.8), we obtain $m_{k}=\hat{m}_{k}$.

Note that for $k$ odd, (3.7) only implies that $Q(\ell) \in \mathbf{N}_{-k+1}$ and that

$$
\ell^{k+1}\left(Q(\ell)-\gamma+\sum_{i=0}^{k-1} \frac{m_{i}}{\ell^{i+1}}\right)+\hat{m}_{k}=o(1)
$$

for $\ell=i y \rightarrow \infty$. If, in this case, the function $\sigma(t)$ in (2.3) has support in $[0, \infty)$, it follows from (3.8) that $Q(\ell) \in \mathbf{N}_{-k}$ and $\hat{m}_{k}=m_{k}$. Moreover, then the function in the left side of (3.7) belongs to $\mathbf{N}_{1}$. In general, for $k$ odd, the function in the left side of (3.7) does not belong to $\mathbf{N}_{1}$ and $\hat{m}_{k}$ in (3.9) cannot be interpreted as an absolutely convergent moment. We give an example for $k=1$.

Example. Let $\sigma(t)$ be a nondecreasing function on $\mathbb{R}$ such that

$$
\int_{\mathbb{R}} d \sigma(t)<\infty, \quad \int_{\mathbb{R}}|t| d \sigma(t)=\infty
$$

and for which the function

$$
F(\ell)=\int_{\mathbb{R}} \frac{1+t \ell}{t-\ell} d \sigma(t)
$$


belongs to $\mathbf{N} \backslash \mathbf{N}_{1}$, while $\lim _{y \rightarrow \infty} F(i y)=0$. The essential part in the construction of such a function is that the support of $\sigma(t)$ is unbounded in each direction; cf. [7]. Clearly, the function

$$
H(\ell)=\int_{\mathbb{R}} \frac{t \ell}{t-\ell} d \sigma(t)
$$

also belongs to $\mathbf{N} \backslash \mathbf{N}_{1}$ and $\lim _{y \rightarrow \infty} H(i y)=0$. Now define

$$
m_{0}=\int_{\mathbb{R}} d \sigma(t), \quad Q(\ell)=-\frac{m_{0}}{\ell}+\frac{H(\ell)}{\ell^{2}} .
$$

Then $Q(\ell)$ has the representation (2.3) with $\gamma=0, Q(\ell) \in \mathbf{N}_{0} \backslash \mathbf{N}_{-1}$, and

$$
\ell^{2}\left(Q(\ell)+\frac{m_{0}}{\ell}\right)=o(1), \quad \ell=i y \rightarrow \infty .
$$

A similar example for positive definite functions is due to A. Wintner; see [15].

\section{LineAR FRACTIONAL TRANSFORMATIONS}

In order to see how the class $\mathbf{N}_{-k}, k \in \mathbb{N} \cup\{0\}$, behaves under the linear fractional transformation (2.6), (2.7), we state and prove the following simple lemma.

Lemma 4.1. Let $c_{i}, d_{i}, i=-1,0,1, \ldots, k$, be real numbers satisfying

$$
c_{-1} d_{-1}=-1, \quad \sum_{j=0}^{i} c_{j-1} d_{i-j-1}=0, \quad i=1, \ldots, k+1,
$$

and let $C(\ell)=o(1), \ell \rightarrow \infty$. Then

$$
-\left(c_{-1}+\frac{c_{0}}{\ell}+\cdots+\frac{c_{k}}{\ell^{k+1}}+\frac{C(\ell)}{\ell^{k+1}}\right)^{-1}=d_{-1}+\frac{d_{0}}{\ell}+\cdots+\frac{d_{k}}{\ell^{k+1}}+\frac{D(\ell)}{\ell^{k+1}}
$$

where $D(\ell)=o(1), \ell \rightarrow \infty$. Moreover,

$$
D(\ell)=\frac{1}{\left(c_{-1}\right)^{2}} C(\ell)+O\left(\frac{1}{\ell}\right), \quad \ell \rightarrow \infty .
$$

Proof. It follows from the assumption about the convolution products that

$$
-D(\ell)=\frac{\alpha(\ell)+\delta(\ell) C(\ell)}{\gamma(\ell)+C(\ell)}
$$

where $\alpha(\ell), \gamma(\ell)$, and $\delta(\ell)$ are polynomials of the form

$$
\alpha(\ell)=\sum_{j=0}^{k} a_{j} \ell^{j}, \quad \gamma(\ell)=\sum_{j=0}^{k+1} c_{j-1} \ell^{k-j+1}, \quad \delta(\ell)=\sum_{j=0}^{k+1} d_{j-1} \ell^{k-j+1} .
$$

Hence, we may write

$$
-D(\ell)=\frac{d_{-1}}{c_{-1}} C(\ell)+\frac{\alpha(\ell)}{\gamma(\ell)+C(\ell)}+\left(\frac{\delta(\ell)}{\gamma(\ell)+C(\ell)}-\frac{d_{-1}}{c_{-1}}\right) C(\ell) .
$$

The degrees of $\alpha(\ell)$ and of $c_{-1} \delta(\ell)-d_{-1} \gamma(\ell)$ are at most $k$, so the second term in the right side is $O(1 / \ell)$ and the third term in the right side is $o(1 / \ell)$. 
According to Lemma 4.1, there is a constant $A>0$, such that

$$
\frac{|\operatorname{Im} D(i y)|}{y} \leq \frac{1}{\left(c_{-1}\right)^{2}} \frac{|\operatorname{Im} C(i y)|}{y}+\frac{A}{y^{2}}, \quad y \geq 1 .
$$

Hence, if $C(\ell) \in \mathbf{N}_{1}$, then $|\operatorname{Im} D(i y)| / y$ is integrable over $[1, \infty)$. If, in addition, $D(\ell) \in \mathbf{N}$, it therefore automatically belongs to $\mathbf{N}_{1}$.

Theorem 4.2. Assume that the function $Q(\ell)$ belongs to $\mathbf{N}_{-k}$ for some $k \in \mathbb{N} \cup\{0\}$. Then

(i) $Q_{\tau}(\ell) \in \mathbf{N}_{-k}$ for $1 / \tau+\gamma \neq 0$,

(ii) $Q_{\tau}(\ell)-\beta \ell \in \mathbf{N}_{-k+2}$ for $1 / \tau+\gamma=0$.

Proof. Without loss of generality we may assume that $\gamma=0$, so that the exceptional value of $\tau$ corresponds to $\tau=\infty$. Due to (2.6) and (2.7), it suffices to show that

$$
-\frac{1}{\frac{1}{\tau}+Q(\ell)} \in \mathbf{N}_{-k} \text { for } \tau \in \mathbb{R} \backslash\{0\},
$$

and

$$
-\frac{1}{Q(\ell)}-\frac{1}{m_{0}} \ell \in \mathbf{N}_{-k+2} \text { for } \tau=\infty .
$$

For the formulation of (4.2) we used that $\beta$ in (2.8) satisfies $\beta=(\operatorname{Im} Q(\mu))^{2} / m_{0}$ when $\gamma=0$; see [8]. As the function $Q(\ell)$ belongs to $\mathbf{N}_{-k}$ for some $k \in \mathbb{N} \cup\{0\}$, it follows from Theorem 3.2 and the assumption $\gamma=0$ that

$$
Q(\ell)=-\sum_{i=0}^{k} \frac{m_{i}}{\ell^{i+1}}+\frac{C(\ell)}{\ell^{k+1}},
$$

where $C(\ell)=o(1), \ell \rightarrow \infty$, and $C(\ell)$ belongs to $\mathbf{N}_{1}$ when $k$ is odd.

We now prove (4.1) for $\tau \in \mathbb{R} \backslash\{0\}$. From (4.3) and Lemma 4.1 we obtain with real numbers $\hat{m}_{i}, i=0, \ldots, k$, the asymptotic expansion

$$
-\frac{1}{\frac{1}{\tau}+Q(\ell)}=-\tau-\sum_{i=0}^{k} \frac{\hat{m}_{i}}{\ell^{i+1}}+\frac{D(\ell)}{\ell^{k+1}},
$$

where $D(\ell)=\tau^{2} C(\ell)+O(1 / \ell)=o(1), \ell \rightarrow \infty$. It follows from Theorem 3.3 and the asymptotic estimate of $D(\ell)$ that (4.1) holds.

Next we prove (4.2). The statement for $k=0$ is obvious, so assume that $k \geq 1$. From (4.3) and Lemma 4.1 (with $k$ instead of $k+1$ ) we obtain with real numbers $\hat{m}_{i}, i=1, \ldots, k$, the asymptotic expansion

$$
-\frac{1}{\ell Q(\ell)}=\frac{1}{m_{0}}-\sum_{i=1}^{k} \frac{\hat{m}_{i}}{\ell^{i}}+\frac{D(\ell)}{\ell^{k}},
$$

with $D(\ell)=C(\ell) / m_{0}^{2}+O(1 / \ell), \ell \rightarrow \infty$. Hence

$$
-\frac{1}{Q(\ell)}-\frac{1}{m_{0}} \ell=-\sum_{i=1}^{k} \frac{\hat{m}_{i}}{\ell^{i-1}}+\frac{D(\ell)}{\ell^{k-1}} .
$$

Again we apply Theorem 3.3 and the asymptotic estimate of $D(\ell)$. Hence, (4.2) holds for $k=1$ and for $k \geq 2$. 
Let $Q(\ell) \in \mathbf{N}_{-k}$ for some $k \in \mathbb{N} \cup\{0\}$. Assume that $1 / \tau+\gamma \neq 0$. Then it follows from Theorems 3.2 and 4.2 that

$$
Q_{\tau}(\ell)=\gamma(\tau)-\sum_{i=0}^{k} \frac{m_{i}(\tau)}{\ell^{i+1}}+o\left(\frac{1}{\ell^{k+1}}\right), \quad \ell \rightarrow \infty
$$

where $\gamma(\tau)=\lim _{y \rightarrow \infty} Q_{\tau}(i y)$ and $m_{i}(\tau), i=0, \ldots, k$, are the corresponding moments of $Q_{\tau}(\ell)$. Now assume that $1 / \tau+\gamma=0$. For $k=0$ the function $Q_{\tau}(\ell)-\beta \ell$ belongs to $\mathbf{N}$, where $\beta$ is given by (2.8). For $k \geq 1$ it belongs to $\mathbf{N}_{1}$, in which case

$$
\gamma(\tau)=\lim _{y \rightarrow \infty}\left(Q_{\tau}(i y)-i \beta y\right)
$$

is a real number. For $k \geq 2$ the function $Q_{\tau}(\ell)-\beta \ell$ belongs to $\mathbf{N}_{-k+2}$, and it follows from Theorems 3.2 and 4.2 that

$$
Q_{\tau}(\ell)-\beta \ell=\gamma(\tau)-\sum_{i=0}^{k-2} \frac{m_{i}(\tau)}{\ell^{i+1}}+o\left(\frac{1}{\ell^{k-1}}\right), \quad \ell \rightarrow \infty,
$$

where $\gamma(\tau)$ is given by (4.5) and $m_{i}(\tau), i=0, \ldots, k-2$, are the moments of $Q_{\tau}(\ell)-\beta \ell$. The constants $\beta$ and $\gamma(\tau)$ and the moments in (4.4) and in (4.6) can be expressed in terms of the corresponding data of the expansion (3.5) of $Q(\ell)$.

Corollary 4.3. Assume that the function $Q(\ell)$ belongs to $\mathbf{N}_{-k}, k \in \mathbb{N} \cup\{0\}$.

For $1 / \tau+\gamma \neq 0$ and $\tau \in \mathbb{R}$, the constant $\gamma(\tau)$ is given by

$$
\gamma(\tau)=\frac{\gamma-\tau(\operatorname{Im} Q(\mu))^{2}}{1+\tau \gamma}
$$

and the moments $m_{i}(\tau), i=0, \ldots, k$, in (4.4) are given by

$$
\begin{aligned}
& \left(\begin{array}{c}
m_{0}(\tau) \\
m_{1}(\tau) \\
\vdots \\
m_{k-1}(\tau) \\
m_{k}(\tau)
\end{array}\right)=\frac{1+(\tau \operatorname{Im} Q(\mu))^{2}}{(1+\tau \gamma)^{2}} \\
& \left(\begin{array}{ccccc}
1 & 0 & \cdots & 0 & 0 \\
-\frac{\tau m_{0}}{1+\tau \gamma} & 1 & & & 0 \\
\vdots & \ddots & \ddots & & \vdots \\
-\frac{\tau m_{k-2}}{1+\tau \gamma} & & \ddots & 1 & 0 \\
-\frac{\tau m_{k-1}}{1+\tau \gamma} & -\frac{\tau m_{k-2}}{1+\tau \gamma} & \cdots & -\frac{\tau m_{0}}{1+\tau \gamma} & 1
\end{array}\right)^{-1}\left(\begin{array}{c}
m_{0} \\
m_{1} \\
\vdots \\
m_{k-1} \\
m_{k}
\end{array}\right) .
\end{aligned}
$$

The case $1 / \tau+\gamma \neq 0$ and $\tau=\infty$ is obtained as a limiting case of (4.7) and (4.8).

For $1 / \tau+\gamma=0$ and $\tau \in \mathbb{R}$, the constant $\beta$ is given by

$$
\beta=\frac{1+(\tau \operatorname{Im} Q(\mu))^{2}}{\tau^{2} m_{0}},
$$

and when $k \geq 1$, the constant $\gamma(\tau)$ is given by

$$
\gamma(\tau)=\frac{1}{\tau}-\frac{1+(\tau \operatorname{Im} Q(\mu))^{2}}{\tau^{2}} \frac{m_{1}}{m_{0}^{2}}
$$


Moreover, when $k \geq 2$ the moments $m_{i}(\tau), i=0, \ldots, k-2$, in (4.6) are given by

$$
\begin{gathered}
\left(\begin{array}{c}
m_{0}(\tau) \\
m_{1}(\tau) \\
\vdots \\
m_{k-3}(\tau) \\
m_{k-2}(\tau)
\end{array}\right)=\frac{1+(\tau \operatorname{Im} Q(\mu))^{2}}{\tau^{2} m_{0}} \\
\qquad\left(\begin{array}{ccccc}
m_{0} & 0 & \cdots & 0 & 0 \\
m_{1} & m_{0} & & & 0 \\
\vdots & \ddots & \ddots & & \vdots \\
m_{k-3} & & \ddots & m_{0} & 0 \\
m_{k-2} & m_{k-3} & \cdots & m_{1} & m_{0}
\end{array}\right) \quad\left(\begin{array}{c}
m_{2}-\frac{m_{1}}{m_{0}} m_{1} \\
m_{3}-\frac{m_{1}}{m_{0}} m_{2} \\
\vdots \\
m_{k-1}-\frac{m_{1}}{m_{0}} m_{k-2} \\
m_{k}-\frac{m_{1}}{m_{0}} m_{k-1}
\end{array}\right)
\end{gathered}
$$

The case $\gamma=0$ and $\tau=\infty$ is obtained as a limiting case of (4.9), (4.10) and (4.11).

Proof. From (2.6) and (2.7) we obtain

$$
\begin{gathered}
Q_{\tau}(\ell)-Q(\ell)+\tau Q(\ell) Q_{\tau}(\ell)+\tau(\operatorname{Im} Q(\mu))^{2}=0, \quad \tau \in \mathbb{R}, \\
Q(\ell) Q_{\infty}(\ell)+(\operatorname{Im} Q(\mu))^{2}=0 .
\end{gathered}
$$

We will substitute the asymptotic expansions (3.5) for $Q(\ell)$ and (4.4) or (4.6) for $Q_{\tau}(\ell)$ in (4.12) and (4.13), and calculate the coefficients of the powers of $\ell$.

For $1 / \tau+\gamma \neq 0$ and $\tau \in \mathbb{R}$ we use the expansion (4.4) for $Q_{\tau}(\ell)$ in (4.12). The coefficient of $\ell^{0}$ gives (4.7), and the coefficient of $\ell^{-1}$ gives

$$
m_{0}(\tau)=\frac{1-\tau \gamma(\tau)}{1+\tau \gamma} m_{0}=\frac{1+(\tau \operatorname{Im} Q(\mu))^{2}}{(1+\tau \gamma)^{2}} m_{0}
$$

Moreover, the coefficients of $\ell^{-i-1}, i=1, \ldots, k$, give

$$
(1+\tau \gamma) m_{i}(\tau)=m_{i}(1-\tau \gamma(\tau))+\tau\left(m_{0} m_{i-1}(\tau)+\cdots+m_{i-1} m_{0}(\tau)\right) .
$$

This leads to (4.8). For $1 / \tau+\gamma \neq 0$ and $\tau=\infty$, we use the expansion (4.4) for $Q_{\tau}(\ell)$ in (4.13) and obtain the limiting case of (4.7) and (4.8) as $\tau \rightarrow \infty$.

For $1 / \tau+\gamma=0$ we substitute the expansion (4.6) in (4.12). Note that the coefficient of $\ell$ is automatically 0 . The coefficient of $\ell^{0}$ gives (4.9) (cf. [8]), and the coefficient of $\ell^{-1}$ gives $1-\tau \gamma(\tau)=\tau m_{1} \beta / m_{0}$, so that (4.10) follows. Similarly, the coefficients of $\ell^{-i-1}$ then give

$$
m_{0} m_{i-1}(\tau)+\cdots+m_{i-1} m_{0}(\tau)=\beta\left(m_{i+1}-\frac{m_{1}}{m_{0}} m_{i}\right),
$$

for $i=1, \ldots, k-2$. Moreover, the identity also holds for $i=k-1$. This leads to (4.11). For $\gamma=0$ and $\tau=\infty$, we use the expansion (4.6) for $Q_{\tau}(\ell)$ in (4.13) and obtain the limiting case of (4.9), (4.10) and (4.11) as $\tau \rightarrow \infty$.

Let $H(\ell)$ be a Nevanlinna function with $\beta=\lim _{y \rightarrow \infty} \operatorname{Im} H(i y) / y>0$. We have seen that $H(\ell)-\beta \ell$ belongs to $\mathbf{N}$. Define the function $Q(\ell)$ by

$$
Q(\ell)=-\frac{|H(\mu)|^{4}}{(\operatorname{Im} H(\mu))^{2} H(\ell)} .
$$

Clearly, $Q(\ell) \in \mathbf{N}_{0}$ and $\lim _{y \rightarrow \infty} Q(i y)=0$. Hence, $H(\ell)=Q_{\infty}(\ell)$ is the exceptional function corresponding to the exceptional value $\tau=\infty$ of $Q(\ell)$; cf. [9]. 
Theorem 4.4. Let $H(\ell)$ belong to $\mathbf{N}$ with $\beta=\lim _{y \rightarrow \infty} \operatorname{Im} H(i y) / y>0$. Assume that $H(\ell)-\beta \ell$ belongs to $\mathbf{N}_{-k+2}$ for some $k \in \mathbb{N} \cup\{0\}$. Then the function $Q(\ell)$ in (4.14) belongs to $\mathbf{N}_{-k}$.

Proof. It is sufficient to assume that $k \geq 1$. Then

$$
H(\ell)-\beta \ell=\gamma-\sum_{i=0}^{k-2} \frac{m_{i}}{\ell^{i+1}}+\frac{C(\ell)}{\ell^{k-1}},
$$

where $\gamma=\lim _{y \rightarrow \infty}(Q(i y)-i \beta y)$ and $m_{i}, i=0, \ldots, k-2$, are the moments of $H(\ell)-\beta \ell$ (absent for $k=1$ ). Moreover, if $k$ is odd, the function $C(\ell)$ belongs to $\mathbf{N}_{1}$. Therefore, by Lemma 4.1 with $k+1$ replaced by $k$, we find real numbers $d_{-1}, \ldots, d_{k-1}$ and a function $D(\ell)=o(1), \ell \rightarrow \infty$, such that

$$
-\frac{1}{H(\ell)}=\frac{1}{\ell}\left(-\frac{1}{\beta+\frac{\gamma}{\ell}-\sum_{i=0}^{k-2} \frac{m_{i}}{\ell^{2+2}}+\frac{C(\ell)}{\ell^{k}}}\right)=\sum_{i=0}^{k} \frac{d_{i-1}}{\ell^{i+1}}+\frac{D(\ell)}{\ell^{k+1}},
$$

with $D(\ell)$ in $\mathbf{N}_{1}$ when $k$ is odd. Hence $Q(\ell) \in \mathbf{N}_{-k}$ by Theorem 3.3.

The relation between the data for the functions $H(\ell)-\beta \ell$ and $Q(\ell)$ may be recovered from Corollary 4.3 by inversion of the case $\gamma=0$ and $\tau=\infty$; cf. [10, Proposition 6.5] for a special case.

\section{RANK ONE PERTURBATIONS}

Let $A$ be a selfadjoint relation in a Hilbert space $\mathfrak{H}$. For $\mu \in \mathbb{C} \backslash \mathbb{R}$ we choose a nontrivial element $\chi(\mu) \in \mathfrak{H}$ and define

$$
\chi(\ell)=\left(I+(\ell-\mu)(A-\ell)^{-1}\right) \chi(\mu) .
$$

Let $S$ be the restriction of $A$ given by

$$
S=\{\{f, g\} \in A:[g-\ell f, \chi(\bar{\ell})]=0\} .
$$

Clearly, this definition is independent of $\ell \in \mathbb{C} \backslash \mathbb{R}$, and $S$ is a closed symmetric relation with defect numbers $(1,1)$. The relation $S$ is completely nonselfadjoint if and only if $\mathfrak{H}=\overline{\operatorname{span}}\{\chi(\ell): \ell \in \mathbb{C} \backslash \mathbb{R}\}$, in which case $S$ is necessarily an operator. A function $Q(\ell)$ is a $Q$-function of $A$ and $S$ if

$$
\frac{Q(\ell)-Q(\lambda)^{*}}{\ell-\bar{\lambda}}=[\chi(\ell), \chi(\lambda)]
$$

Hence, a $Q$-function is determined up to a real constant and belongs to the Nevanlinna class $\mathbf{N}$. If $S$ is completely nonselfadjoint, the $Q$-function uniquely determines, up to isometric isomorphisms, the relation $A$ and its restriction $S$. All selfadjoint extensions $A(\tau), \tau \in \mathbb{R} \cup\{\infty\}$, of $S$ are parametrized by means of Krel̆n's formula

$$
(A(\tau)-\ell)^{-1}=(A-\ell)^{-1}-\chi(\ell) \frac{1}{Q(\ell)+1 / \tau}[\cdot, \chi(\bar{\ell})] .
$$

The $Q$-functions $Q_{\tau}(\ell)$ of $A(\tau), \tau \in \mathbb{R} \cup\{\infty\}$, are related to $Q(\ell)$ via (2.6) and (2.7); see [8].

In the following we assume that $A$ is a selfadjoint operator. The restriction $S$ in (5.1) coincides with (1.2) if and only if $\chi(\ell) \in \operatorname{dom} A$ for some (and hence for all) $\ell \in \rho(A)$. Then $\chi(\ell)=(A-\ell)^{-1} \omega$, and $Q(\ell)$ can be chosen as

$$
Q(\ell)=\left[(A-\ell)^{-1} \omega, \omega\right] .
$$


This choice of $Q(\ell) \in \mathbf{N}_{0}$ gives $\gamma=0$, so that the exceptional value in (2.6) and (2.7) is $\tau=\infty$. The selfadjoint extensions of $S$ in (1.2) are now the rank one perturbations $A(\tau), \tau \in \mathbb{R}$, of $A$ given in (1.1) (cf. [4]), and the exceptional extension $A(\infty)$ in (1.3). If $E(t), t \in \mathbb{R}$, is the spectral family of $A$, and $Q(\ell)$ is given by (2.3) with $\gamma=0$ and $(2.5)$, then $d \sigma(t)=d([E(t) \omega, \omega])$. We denote the polar decomposition of $A$ by $A=U|A|$. The following result is clear.

Theorem 5.1. Let $k \in \mathbb{N} \cup\{0\}$. Then $Q(\ell) \in \mathbf{N}_{-k}$ if and only if $\omega \in \operatorname{dom}|A|^{k / 2}$. In this case, the moments $m_{j}, j=0, \ldots, k$, are given by

$$
m_{j}=\left[A^{j / 2} \omega, A^{j / 2} \omega\right], \quad j \text { even, } \quad m_{j}=\left[U|A|^{j / 2} \omega,|A|^{j / 2} \omega\right], \quad j \text { odd } .
$$

Note that if $A \in \mathbf{L}(\mathfrak{H})$, then each $\omega \in \mathfrak{H}$ has the property that $\omega \in \operatorname{dom}|A|^{k / 2}$, for all $k \in \mathbb{N} \cup\{0\}$. In particular this applies when the closed symmetric operator $S$ is bounded and, consequently, $A(\tau) \in \mathbf{L}(\mathfrak{H}), \tau \in \mathbb{R}$; see also [17].

Theorem 5.2. Assume that the $Q$-function $Q(\ell)$ of $S$ and $A$ belongs to $\mathbf{N}_{-k}$ for some $k \in \mathbb{N} \cup\{0\}$. Then

$$
\operatorname{dom}|A(\tau)|^{k / 2+1}=\operatorname{dom}|A|^{k / 2+1}, \quad \tau \in \mathbb{R} \cup\{\infty\}, 1 / \tau+\gamma \neq 0 .
$$

Proof. The statement is true for $k=0,1,2$; cf. [11]. We proceed by induction. Let $g \in \operatorname{dom}|A(\tau)|^{k / 2+1}$, so that $g=(A(\tau)-\ell)^{-1} f$ for some $f \in \operatorname{dom}|A(\tau)|^{k / 2}$. By Kreĭn's formula

$$
g=(A-\ell)^{-1} f-\frac{[f, \chi(\bar{\ell})]}{1 / \tau+Q(\ell)} \chi(\ell) .
$$

Since $Q(\ell) \in \mathbf{N}_{-k}$, Theorem 5.1 shows that $\chi(\ell)=(A-\ell)^{-1} \omega \in \operatorname{dom}|A|^{k / 2+1}$. Moreover, $\mathbf{N}_{-k} \subset \mathbf{N}_{-k+2}$, and since $1 / \tau+\gamma \neq 0$, we conclude by an induction argument that

$$
f \in \operatorname{dom}|A(\tau)|^{k / 2}=\operatorname{dom}|A|^{k / 2} .
$$

Hence, $(A-\ell)^{-1} f \in \operatorname{dom}|A|^{k / 2+1}$. It follows from (5.3) that $g \in \operatorname{dom}|A|^{k / 2+1}$ and therefore

$$
\operatorname{dom}|A(\tau)|^{k / 2+1} \subset \operatorname{dom}|A|^{k / 2+1}, \quad 1 / \tau+\gamma \neq 0 .
$$

According to Theorem 4.2, $A$ and $A(\tau), 1 / \tau+\gamma \neq 0$, both have a $Q$-function belonging to $\mathbf{N}_{-k}$, so the reverse inclusion follows by symmetry.

Theorem 5.3. Let $S$ be a closed symmetric operator in $\mathfrak{H}$ with defect numbers $(1,1)$ and let $\alpha \geq 0, k \in \mathbb{N} \cup\{0\}$. If for two different selfadjoint operator extensions $A_{1}$ and $A_{2}$ of $S$ the inclusion

$$
\operatorname{dom}\left|A_{1}\right|^{k / 2+1} \supset \operatorname{dom}\left|A_{2}\right|^{k / 2+1+\alpha}
$$

is satisfied, then for all but one selfadjoint extension $A(\tau)$ of $S$ we have

$$
\operatorname{dom}|A(\tau)|^{k / 2+1}=\operatorname{dom}\left|A_{1}\right|^{k / 2+1} .
$$

Moreover, the $Q$-functions of these extensions of $S$ all belong to $\mathbf{N}_{-k}$.

Proof. The statements hold for $k=0,1,2$; cf. [11]. Let $R_{1}(\ell)$ and $R_{2}(\ell)$ be the resolvent operators of $A_{1}$ and $A_{2}$, respectively. Let $h \in \operatorname{dom}\left|A_{2}\right|^{k / 2+\alpha}, k \geq 2$, be 
such that $[h, \chi(\bar{\ell})] \neq 0$. By Kreı̀'s formula

$$
\frac{[h, \chi(\bar{\ell})]}{1 / \tau+Q(\ell)} \chi(\ell)=R_{1}(\ell) h-R_{2}(\ell) h, \quad h \in \mathfrak{H}, \quad \tau \neq 0 .
$$

Since $R_{1}(\ell) h \in \operatorname{dom} A_{1}$ and

$$
R_{2}(\ell) h \in \operatorname{dom}\left|A_{2}\right|^{k / 2+1+\alpha} \subset \operatorname{dom}\left|A_{1}\right|^{k / 2+1} \subset \operatorname{dom} A_{1},
$$

it follows from (5.4) and the selection of $h$ that $\chi(\ell) \in \operatorname{dom} A_{1}$. Hence, we may write $\chi(\ell)=\left(A_{1}-\ell\right)^{-1} \omega$ for some $\omega \in \mathfrak{H}$. Since $A_{1}$ and $A_{2}$ both are operator extensions of $S$, Theorems 4.2, 5.1, and 5.2 imply that $\operatorname{dom} A_{1}=\operatorname{dom} A_{2}$. Hence, $h \in \operatorname{dom} A_{1}$, and thus (5.4) shows that $\chi(\ell) \in \operatorname{dom} A_{1}^{2}$ or $\omega \in \operatorname{dom} A_{1}$. Repeating this argument, we finally observe that, in fact,

$$
\chi(\ell)=\left(A_{1}-\ell\right)^{-1} \omega \in \operatorname{dom}\left|A_{1}\right|^{k / 2+1},
$$

or, equivalently, $\omega \in \operatorname{dom}\left|A_{1}\right|^{k / 2}$. According to Theorem 5.1 the $Q$-function $Q(\ell)$ of $A_{1}$ and $S$ belongs to $\mathbf{N}_{-k}$. Now apply Theorems 5.2 and 4.2 .

\section{REFERENCES}

[1] N.I. Achieser, The classical moment problem and some related questions in analysis, Fizmatgiz, Moscow, 1961 (Russian) (English translation: Oliver and Boyd, Edinburgh, and Hafner, New York, 1965). MR 32:1518

[2] S. Albeverio and P. Kurasov, "Rank one perturbations of not semibounded operators", Integral Equations Operator Theory 27 (1997), 379-400. CMP 97:10

[3] E.A. Coddington and H.S.V. de Snoo, "Positive selfadjoint extensions of positive symmetric subspaces", Math. Z., 159 (1978), 203-214. MR 58:17936

[4] W.F. Donoghue, Monotone matrix functions and analytic continuation, Springer-Verlag, Berlin-Heidelberg-New York, 1974. MR 58:6279

[5] F. Gesztesy and B. Simon, "Rank one perturbations at infinite coupling", J. Functional Analysis, 128 (1995), 245-252. MR 95m:47014

[6] S. Hassi, M. Kaltenbäck, and H.S.V. de Snoo, "Triplets of Hilbert spaces and Friedrichs extensions associated with the subclass $\mathbf{N}_{1}$ of Nevanlinna functions", J. Operator Theory, 37 (1997), 155-181. CMP 97:09

[7] S. Hassi, M. Kaltenbäck, and H.S.V. de Snoo, "A characterization of semibounded selfadjoint operators", Proc. Amer. Math. Soc. 125 (1997), 2681-2692. CMP 97:12

[8] S. Hassi, H. Langer, and H.S.V. de Snoo, "Selfadjoint extensions for a class of symmetric operators with defect numbers $(1,1)$ ", Topics in Operator Theory, Operator Algebras and Applications (Timisoara, 1994), Romanian Acad., Bucharest, 1995, pp. 115-145. CMP 97:04

[9] S. Hassi and H.S.V. de Snoo, "On some subclasses of Nevanlinna functions", Zeitschrift für Analysis und ihre Anwendungen, 15 (1996), 45-55. MR 96k:47044

[10] S. Hassi and H.S.V. de Snoo, "One-dimensional graph perturbations of selfadjoint relations", Ann. Acad. Sci. Fenn., Series A.I. Math., 22 (1997), 123-164. CMP 97:07

[11] S. Hassi and H.S.V. de Snoo, "Nevanlinna functions, perturbation formulas, and triplets of Hilbert spaces", Math. Nachr., (to appear).

[12] I.S. Kac, "On integral representations of analytic functions mapping the upper half-plane onto a part of itself", Uspehi Mat. Nauk, 11 (1956), no. 3 (69), 139-144 (Russian). MR 18:293C

[13] I.S. Kac and M.G. Kreĭn, " $R$-functions-analytic functions mapping the upper halfplane into itself", Supplement I to the Russian edition of F.V. Atkinson, Discrete and continuous boundary problems, Mir, Moscow, 1968 (Russian) (English translation: Amer. Math. Soc. Transl., (2) 103 (1974), 1-18).

[14] A. Kiselev and B. Simon, "Rank one perturbations with infinitesimal coupling", J. Functional Analysis, 130 (1995), 345-356. MR 96e: 47012

[15] E. Lukacs, Characteristic functions, Charles Griffin \& Company Limited, London, 1960. MR 24:A1392 
[16] B. Simon, "Spectral analysis of rank one perturbations and applications", in J. Feldman, R. Froese, and L.M. Rosen (editors), Proceedings on Mathematical Quantum Theory II: Schrödinger operators, CRM Proceedings and Lecture Notes, Vol. 8, Amer. Math. Soc., Providence, R.I., 1995. MR 97c: 47008

[17] A.V. Štraus, "Generalized resolvents of bounded symmetric operators", Funkts. Anal., 27 (1987), 187-196 (Russian). MR 93d:47050

Department of Statistics, University of Helsinki, PL 54, 00014 Helsinki, Finland

E-mail address: hassi@cc.helsinki.fi

Department of Mathematics, University of Groningen, Postbus 800, 9700 AV GroninGen, Nederland

E-mail address: desnoo@math.rug.nl 